\title{
Low-Cost Indoor Localization Using Sound Spectrum of Light Fingerprints
}

\author{
Chung-Wen Hung \\ Department of Electrical Engineering, National Yunlin University of Science and Technology. Douliou, Taiwan
}

Hiroyuki Kobayashi

Osaka Institute of Technology, Osaka, Japan

Jun-Rong Wu

Department of Electrical Engineering. National Yunlin University of Science and Technology. Douliou, Taiwan

Chau-Chung Song

Department of Electrical Engineering, Department of Aeronautical Engineering, Taiwan

E-mail:wenhung@yuntech.edu.tw,hiroyuki.kobayashi@oit.ac.jp_m10812085@yuntech.edu.tw,ccsong@gs.nfu.edu.tw

\begin{abstract}
A low-cost indoor localization system using sound spectrum of light fingerprint is proposed in this paper, an artificial intelligence, AI, algorithm will be implemented in a low-cost micro-control unit, MCU, to perform the localization. The unit, complex and tiny differences of the light fingerprints are caused by the different characteristics of the discrete components used in lighting devices. To reduce the memory size for low-cost MCU, only sound spectrum of light fingerprint is used to identification the lighting device. Moreover, the grid search also is used to optimize the hyperparameters to compact the AI model. The system architecture and algorithm development are discussed in this paper, and the experimental results will be present to show the proposed system workable
\end{abstract}

Keywords: light fingerprint, machine learning, indoor, localization

\section{Introduction}

Indoor localization is an issue in may application, such as automated guided vehicle, smart building. However, the traditional Positioning method, Global Positioning System (GPS), is not workable indoors. Many indoor localization literatures based localization caught eyes due to infrastructure-less character. However, how to modularize the localization method is other key point for using the technology in indoor equipment.

A mobile phone was used to sense the light and convert it to the received light strength (RLS). This RLS would be compared with other RLSs on the map, then the position was located by the similar RLS [1]. Four LEDs placed on the ceiling were used to transmit the encoded and modulated optical signals with position coordinates, and the signal received from photodiode receiver was analyzed to calculated the position [2]. In [3], light signals were acquired at high frequency, up to 1 megasamples/second, then mapped into the frequency domain by Fast Fourier Transform (FFT). The k-Nearest Neighbor $(\mathrm{KNN})$ and Convolutional Neural Net $(\mathrm{CNN})$ classifiers were discussed. The hardware system comprised of a high-frequency light sensor, analog-to-digital converter (ADC) scope, and Raspberry-PI processor to the performance of the classifiers. [4] used the microphone build in the mobile phone to sampling the light signal sensed by a photodiode (PD), then the sampling were sent to the cloud server through the network. The FFT preprocessing and 1-dimensional convolutional neural network CNN were calculation on the server, and classification is performed. [1] and [2] used other characteristics for localization, and never mention about realization. [3] achieved the modularized target, and the proposed system included an extra high sampling rate ADC. The method proposed in [4] only needed the acoustic frequency spectrum of the light, it means lower sampling frequency requirement. Then, all the proposed localization algorithm was performed in cloud sever. The proposed method in this paper is similar to [4] with extension and modification, and how to realize the method into a module will be detailed.

\section{Data Acquisition}

The text is to be typeset in $10 \mathrm{pt}$ Times Roman, single spaced with interline spacing of $13 \mathrm{pt}$. Text area (excluding running title) is 6.75 inches across and 8.8 inches deep. Final pagination and insertion of running titles will be done by the publisher, so make sure that no page numbers are given in your paper and only the running titles provided in this template (authors' names and paper title) are used. 


\section{A Data Acquisition and feature spectrum extraction}

The analog to digital converter, ADC, build in MCU is used to sample the time domain data. First, higher sampling rate and the larger number of sampling points is selected to confirm the feature frequency, first. Then, the suitable sampling rate and point will be optimized, means to minimize these two number but keep the feature. The preprocessor is discrete Fourier transform, DFT, to present the frequency feature of the sampling data. The formula is show in (1), $X[$.$] and x[$.$] represent respectively$ the frequency and time domain data; $k$ and $n$ denotes the $\mathrm{k}$-th point frequency and $\mathrm{n}$-th point sampling data, and $\mathrm{N}$ is the totally sampling points. The frequency resolution, $\Delta f$, of FFT is as (2), here, $f_{s}$ indicates the sampling rate (frequency). The typical FFT results of two fluorescent lamps are shown in Fig. 1 and 2, the 16,384 points time domain data sampled at $65536 \mathrm{~Hz}$.

$$
\begin{gathered}
X[k]=\sum_{n=0}^{N-1}\left(x[n] \times e^{-i \frac{2 \pi n k}{N}}\right), \\
k=0,1, \ldots, N-1 \\
\Delta f=\frac{f_{s}}{N}
\end{gathered}
$$

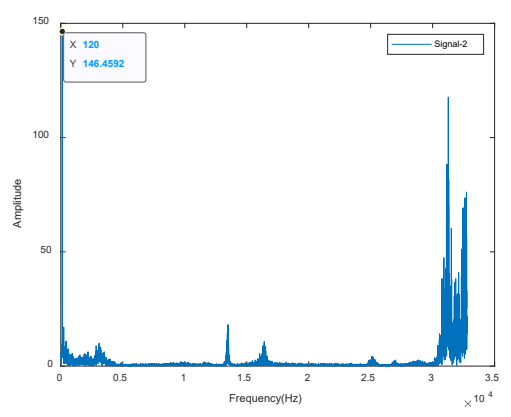

Fig. 1 The spectrum of the fluorescent tubes 1

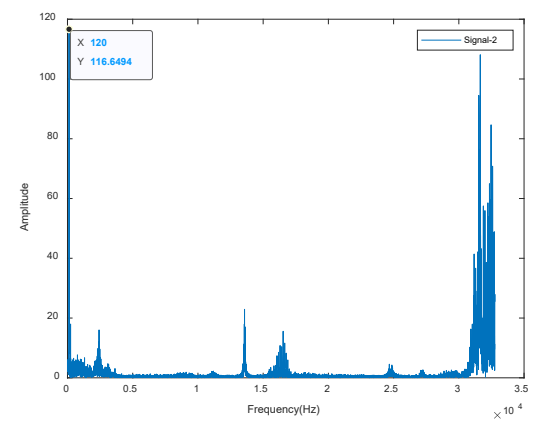

Fig. 2 The spectrum of the fluorescent lamp 2
The spectrums of these two fluorescent lamps are different, and the most features of the spectrum locate at low frequency interval. Note, here is a huge spike at $120 \mathrm{~Hz}$ no matter lamp 1 or 2 , because fluorescent lights is powered by magnetic ballasts with a frequency of $60 \mathrm{~Hz}$ $(50 \mathrm{~Hz}$ in some country) and lamps flicker with the double frequency. The portion less than $150 \mathrm{~Hz}$ will not be used in localization. In the other side, there are also some peaks around $32 \mathrm{~K} \mathrm{~Hz}$. The portion near Nyquist frequency is also ignored. Only the spectrum from 150 $\mathrm{Hz}$ to $30 \mathrm{~K} \mathrm{~Hz}$ is used to machine learning and localization. The Fig. 3 and 4 are the spectrums of the two lamps in selected frequency range

\section{B Rescaling}

For modularization, the artificial intelligence algorithm will be implemented into an MCU, which calculation and memory capabilities are limited. Smaller node number of input layer means less operation and memory requirement. The reduction of the input nodes but without losing frequency feature is important. As shown in Fig. 3 and 4, the low frequency spectrum contains most information, so frequency rescaling equation will be adopted to cover original FFT data to the characteristic vector[4]. The frequency axis will be rescaled to power of $F_{\text {factor }}$ as shown in (3), and the $F_{\text {factor }}$ is shown as (4). Here, $f_{\max }$ and $f_{\text {min }}$ indicate the frequency range which is used in machine learning, and $N_{F_{-} \text {target }}$ is the target number of reduction. Finally, the amplitude of rescaling spectrum, $A[i]$, is the summation of the amplitude which frequency between two rescaling frequency axis point. (5).

$$
\begin{gathered}
F(i)=\left(F_{\text {factor }}\right)^{i} \\
F_{\text {factor }}=\left(\frac{f_{\text {max }}-f_{\text {min }}}{f_{\text {min }}}\right)^{\frac{1}{N_{-} \text {target }}} \\
\sum_{j}{ }_{F_{\text {factor }}{ }^{i} j * \Delta f \leq F_{\text {factor }}{ }^{i+1}}|X[j]|
\end{gathered}
$$

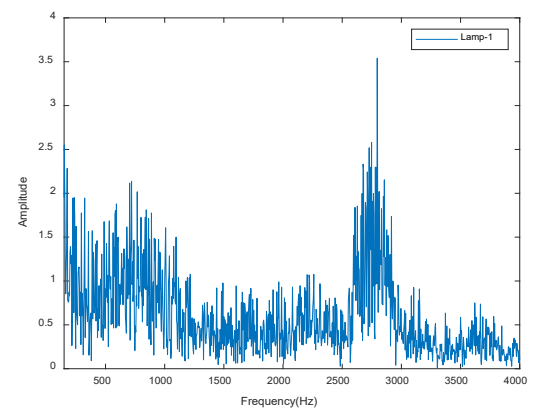

Fig. 3 The spectrum of 150 to $4000 \mathrm{~Hz}$ for the lamp 1 


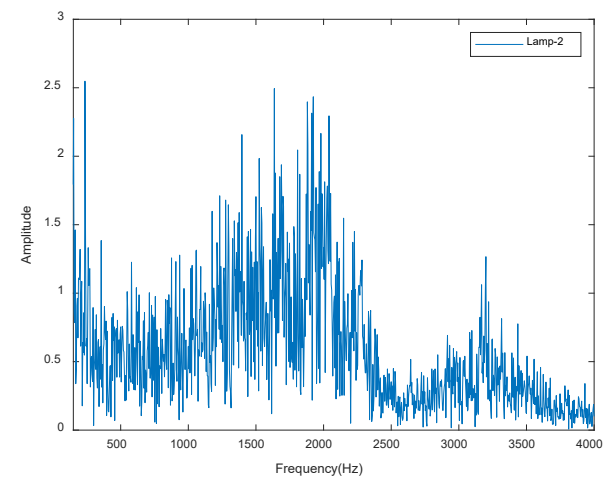

Fig. 4 The spectrum of 150 to $30 \mathrm{k} \mathrm{Hz}$ for the lamp 2

The Fig 5. presents the rescaling results of FFT for the two lamp. Here, $f_{\text {max }}$ and $f_{\min }$ are set to 150 and $30 \mathrm{k}$ $\mathrm{Hz}$, and rescaling points are 400 . The rescaling results keep the original features in low frequency spectrum, and high frequency's is summed into low resolution form. However, the input data are 75 times smaller, this rescaling processing will reduce the requirement of memory and calculation dramatically. In other words, it is very helpful for modularization in a low-cost MCU.

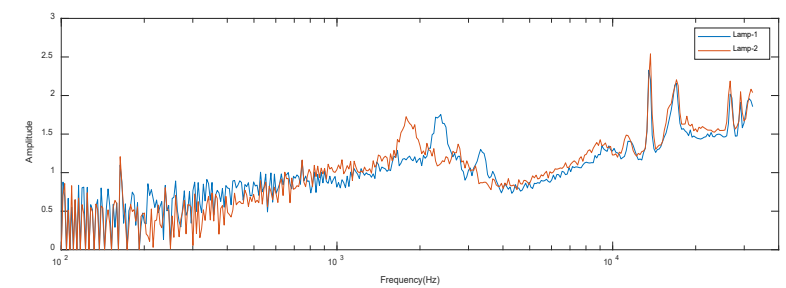

Fig. 5 The rescaling result for the lamp 1 and 2

\section{Identification Algorithm and Grid Search for Hyperparameters}

CNN is a popular algorithm for classification due to the its ability to learn to extract the features, and less parameter memory and calculation requirement is suitable for modularization [5]-[6]. 1-D CNN is adopted in this paper due to the resource limitation in low-cost MCU, no matter memory or calculation speed. The filter kernel in convolution layer is used to extract certain features from rescaling spectrum, and full connected layers perform the classification, but pooling layer is skipped in this application.
Grid search is standard skill to get the hyperparameter combination of the highest accuracy [7], such as CNN Filter Kernel number and size. Different from general grid search which only focus on accuracy, the memory size of parameter is also a key factor for modularization. The grid search items and the range is expressed in table I, here are totally 240 parameter combination.

Table I. The items and parameter of grid search

\begin{tabular}{ll}
\hline Item & Parameter \\
\hline CNN Filter Kernel number & 1,2 \\
CNN Filter Size & $2 、 4 、 8 、 16 、 32 、 64 、$ \\
& $128 、 256$ \\
Activation Function of Fully Connected layer & ReLU、Tanh、Sigmoid \\
Node of Fully Connected layer & $64 、 32 、 16 、 8 、 4$ \\
\hline
\end{tabular}

Localization is designed for eight lamps position, and 100 averaged rescaling data is collected for each lamp for training. To eliminate random errors caused by initial random weights, the three training results for each parameter combination are averaged. The iteration number set to 1000 , and early stopping is adopted to avoid overfitting per training round. The grid search results are sorted by verified accuracy and parameter file size. In the 240 training results for all parameter combination, the top ten results are shown in Table 2.

Table 2. The Top Ten Results Gid Search

\begin{tabular}{|c|c|c|c|c|c|c|c|}
\hline $\begin{array}{l}\text { Train. } \\
\text { Acc. }\end{array}$ & $\begin{array}{l}\text { Train. } \\
\text { Loss }\end{array}$ & $\begin{array}{l}\text { Verified } \\
\text { Acc. }\end{array}$ & $\begin{array}{l}\text { Filter } \\
\text { Kernel }\end{array}$ & $\begin{array}{l}\text { Filter } \\
\text { Size }\end{array}$ & $\begin{array}{l}\text { Act. } \\
\text { Func. }\end{array}$ & $\begin{array}{l}\text { Nodes of } \\
\text { Fully } \\
\text { Connected } \\
\text { Layer }\end{array}$ & $\begin{array}{l}\text { Memory } \\
\text { Size (kB) }\end{array}$ \\
\hline 1 & 0.000577 & 1 & 1 & 256 & ReLU & $32 / 16 / 16$ & 5.96875 \\
\hline 1 & 0.010547 & 1 & 1 & 256 & Tanh & $32 / 16 / 16$ & 5.96875 \\
\hline 1 & 0.051400 & 1 & 1 & 256 & Sigmoid & $132 / 16 / 16$ & 5.96875 \\
\hline 1 & 0.010734 & 1 & 1 & 128 & Tanh & $32 / 16 / 16$ & 9.71875 \\
\hline 1 & 0.048613 & 1 & 1 & 128 & Sigmoid & $132 / 16 / 16$ & 9.71875 \\
\hline 1 & 0.010579 & 1 & 2 & 256 & Tanh & $32 / 16 / 16$ & 10.4687 \\
\hline 1 & 0.085243 & 1 & 2 & 256 & Sigmoid & $132 / 16 / 16$ & 10.4687 \\
\hline 1 & 0.011359 & 1 & 1 & 64 & Tanh & $32 / 16 / 16$ & 11.5937 \\
\hline 1 & 0.002533 & 1 & 1 & 256 & Tanh & $64 / 32 / 32$ & 12.9375 \\
\hline 1 & 0.010598 & 1 & 1 & 256 & Sigmoid & $164 / 32 / 32$ & 12.9375 \\
\hline
\end{tabular}

The parameters of CNN model are detailed in Table 3 , the some parameters are get from the grid search. The settings of fully connected layer number and activation function set to 3 and tanh [4]. The number of output node is eight, its form is likelihood by softmax function. 


\section{Implementation}

The main target in this paper is a low-cost indoor localization module. The RX65N MCU provided by Renesas Electronic Corporation is used to implemented the proposed algorithm. The build-in $2 \mathrm{M}$ bytes of read only memory, ROM, and $640 \mathrm{~K}$ Bytes random access memory, RAM, are enough to install the optimized AI model, and the high-speed operation with 32 bit floating point could support the calculation requirement of FFT.

Table 3 The Items and Parameter of Grid Search

\begin{tabular}{ll}
\hline Item & Parameter \\
\hline CNN Filter Kernel number & 1 \\
CNN Filter Size & $256^{*} 1$ \\
Activation Function of CNN & ReLU \\
Node of Fully Connected layer 1 & 32 \\
Node of Fully Connected layer 2 & 16 \\
Node of Fully Connected layer 3 & 16 \\
Activation Function of Fully Connected layer & Tanh \\
Node number of Output layer & 8 \\
Activation Function of Output layer & SoftMAX \\
\hline
\end{tabular}

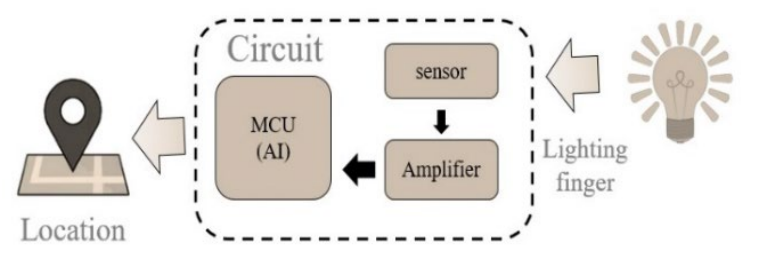

Fig.6 The architecture diagram of system

The proposed system is shown in Fig. 5, it includes a sensor circuit, amplifier block and MCU. The sensor circuit consists of a photodiode and resister, and the amplifier block is implemented with operational amplifier to perform the gain and filter functions. The system hardware is present as Fig. 6. Note, the hardware is only for experimentation but not size-optimization yet.

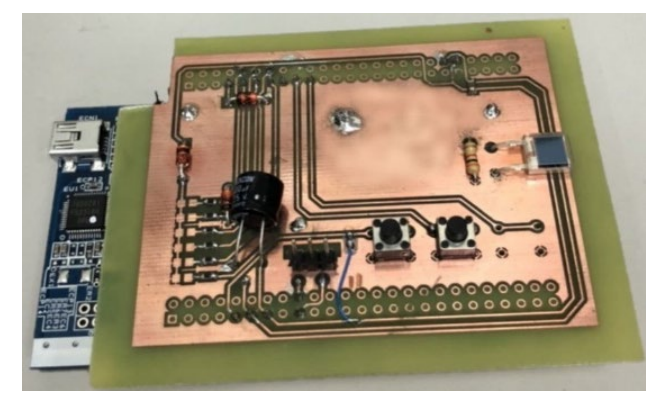

Fig. 7 The photo of proposed hardware
The signal processing flow is dedicated in Fig. 7, moreover, the processing time of every sub processing block is also shown. The longest duration is data acquisition. However, it is necessary, because the data is sampled three times, and there is 16,384 points sampled at $65,536 \mathrm{~Hz}$ every time. The transfer time from time domain data to frequency domain may be shorted if fast MCU. And before localization AI algorithm, the rescaling FFT results of three samples are averaged.

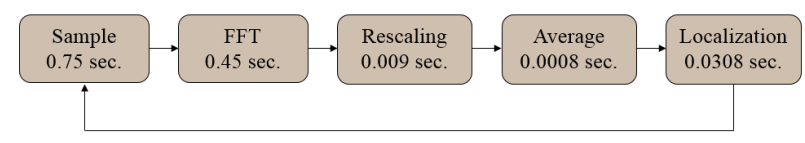

Fig. 8 The signal processing flow is dedicated

\section{Experiment}

\section{A Setup}

The experiment was performed in the EN303 room on third floor of the Engineering sixth hall, National Yunlin University of Science and Technology, as shown in Fig. 8. The proposed module is located about 1.7 meters below lamps. The numbers denote the lamps. The floor plan is revealed in Fig. 9, which also includes the location of lamps, the distance between each lamps and from lamp to hall. Note, the ' $X$ ' marks indicate the location when testing the lamp, and the ' + ' marks shows the position when testing not below the lamps.

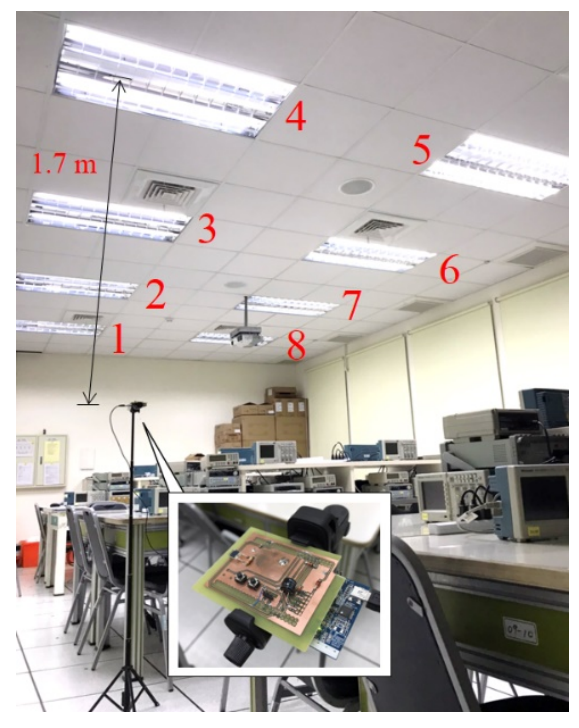

Fig. 9 Experiment in the EN303 room

(C) The 2021 International Conference on Artificial Life and Robotics (ICAROB2021), January 21 to 24, 2021 


\section{B Results}

The estimated likelihoods of classification are shown in Table 4, the proposed module is placed below the lamps which are marked by ' $\mathrm{X}$ ' turn by turn. The number in the table indicates the most likelihood lamp number, and the number in the parentheses shows the estimated likelihood. The experiment ran 10 turns, and every ' $\mathrm{X}$ ' point is tested every turn. The localization accuracy performed $100 \%$. The least likelihood in the table locates at lamp 4 in the turn 8 , however the likelihood still achieved $75.8 \%$, it demonstrated the localization is correct.

The Table 5 present an interesting estimation results, the average likelihood of 10 turns is expressed by percentage, when the proposed module is placed at mark '+' position. The two adjacent likelihoods shown in bold are expected to be biggest two number, because the testing position is located between the two lamps indicated in the first line of table. However, the expectation was correct such as position 9,11 and 13, but incorrect for other points. The reason should be that the sample located the position 9 to 14 never be adopted for AI model training and the estimated likelihood is not the interpolation of any two training points. The future work is to adopt such position sample to machine learning to evaluate the localization effect if the light sampled not below the lighting source.

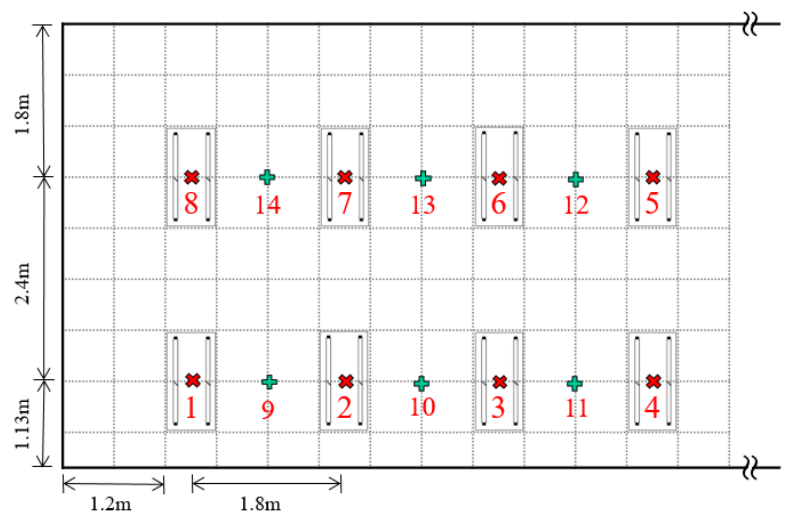

Fig. 10 Floor Experiment in the EN303 room

Table 4. Like hoods of Classification of the Turns

\begin{tabular}{ccccccccc}
\hline Turn Lamp1 Lamp2 Lamp3 Lamp4 Lamp5 Lamp6 Lamp7 Lamp8 \\
\hline $\mathbf{1}$ & $1(98.8)$ & $2(98.9)$ & $3(98.9)$ & $4(97.6)$ & $5(96.2)$ & $6(98.9)$ & $7(98.4)$ & $8(99.1)$ \\
$\mathbf{2}$ & $1(98.6)$ & $2(98.9)$ & $3(97.2)$ & $4(97.6)$ & $5(98.1)$ & $6(98.8)$ & $7(96.5)$ & $8(99.1)$ \\
$\mathbf{3}$ & $1(98.2)$ & $2(98.9)$ & $3(98.2)$ & $4(95.3)$ & $5(98.2)$ & $6(98.8)$ & $7(98.5)$ & $8(99.0)$ \\
$\mathbf{4}$ & $1(98.5)$ & $2(98.9)$ & $3(98.6)$ & $4(93.1)$ & $5(96.9)$ & $6(98.9)$ & $7(98.3)$ & $8(99.1)$ \\
$\mathbf{5}$ & $1(97.4)$ & $2(99.0)$ & $3(91.3)$ & $4(95.1)$ & $5(97.3)$ & $6(98.9)$ & $7(98.5)$ & $8(98.9)$
\end{tabular}

Table 4. (Continued)

\begin{tabular}{rrrrrrrrr}
\hline $\mathbf{6}$ & $1(98.9)$ & $2(99.0)$ & $3(98.9)$ & $4(96.4)$ & $5(98.2)$ & $6(98.8)$ & $7(98.5)$ & $8(99.1)$ \\
$\mathbf{7}$ & $1(98.6)$ & $2(99.0)$ & $3(98.5)$ & $4(94.2)$ & $5(98.2)$ & $6(98.9)$ & $7(98.5)$ & $8(99.1)$ \\
$\mathbf{8}$ & $1(98.8)$ & $2(98.9)$ & $3(96.4)$ & $4(75.8)$ & $5(98.2)$ & $6(98.9)$ & $7(98.5)$ & $8(99.0)$ \\
$\mathbf{9}$ & $1(98.7)$ & $2(98.9)$ & $3(98.5)$ & $4(93.9)$ & $5(98.0)$ & $6(98.8)$ & $7(98.5)$ & $8(99.1)$ \\
$\mathbf{1 0}$ & $1(98.9)$ & $2(98.9)$ & $3(95.0)$ & $4(90.8)$ & $5(98.1)$ & $6(98.8)$ & $7(98.3)$ & $8(98.8)$ \\
\hline
\end{tabular}

Table 5. Likehood Estimation Between Two Lamps

\begin{tabular}{cllllllll}
\hline Pos. & Lamp1 Lamp2 & Lamp3 & Lamp4 & Lamp5 & Lamp6 & Lamp7 & Lamp8 \\
\hline $\mathbf{9}$ & $\mathbf{2 8 . 3 3 3}$ & $\mathbf{2 6 . 8 7 9}$ & 1.589 & 3.464 & 36.366 & 1.612 & 1.506 & 0.251 \\
$\mathbf{1 0}$ & 0.113 & $\mathbf{8 0 . 0 0 2}$ & $\mathbf{7 . 0 9 0}$ & 0.288 & 0.033 & 10.378 & 1.790 & 0.306 \\
$\mathbf{1 1}$ & 0.138 & 0.054 & $\mathbf{3 8 . 1 0 9}$ & $\mathbf{6 0 . 7 9 9}$ & 0.490 & 0.056 & 0.300 & 0.055 \\
$\mathbf{1 2}$ & 0.033 & 0.652 & 0.017 & 0.123 & $\mathbf{0 . 5 0 9}$ & $\mathbf{9 7 . 5 2 1}$ & 0.863 & 0.283 \\
$\mathbf{1 3}$ & 0.011 & 1.321 & 0.046 & 0.267 & 0.757 & $\mathbf{2 2 . 1 3 6}$ & $\mathbf{7 4 . 4 6 0}$ & 1.001 \\
$\mathbf{1 4}$ & 0.080 & 0.034 & 0.004 & 0.193 & 0.657 & 0.976 & $\mathbf{1 . 4 4 3}$ & $\mathbf{9 6 . 6 2 0}$ \\
\hline
\end{tabular}

\section{Conclusion}

In this paper, a low-cost indoor localization system using sound spectrum of light fingerprint is proposed. To use this light fingerprint based localization indoor mobile equipment, modularization is the key consideration. So, the memory and calculation of the selected MCU are limited due to cost. Only the rescaling frequency features between $100 \mathrm{~Hz}$ to $30 \mathrm{k} \mathrm{Hz}$ are used in classification, and a simplified 1-D CNN is performed to classify the lamps. Grid search is adopted to optimize the hyperparameters to find the highest-accuracy and smallest-size model. The experiment shows the system could be implemented in a lost-cost MCU with few components. The on-line tests present the localization accuracy achieve $100 \%$ when the proposed device located below the lamps. However, not as expected, when testing position locates between lamps, the likelihood is not an interpolation of the nearest two lamps'. It may be the future works of this topic.

\section{Acknowledgment}

Part of this work was financially supported by the "Intelligent Recognition Industry Service Research Center" from The Featured Areas Research Center Program within the framework of the Higher Education Sprout Project by the Ministry of Education (MOE) in Taiwan. And it is also supported by Ministry of Science and Technology, Taiwan, under contract MOST 1082221-E-224-045- and 108-2218-E-150-004-. 


\section{References}

[1] Y. Hu et al., "Lightitude: Indoor Positioning Using Ubiquitous Visible Lights and COTS Devices," 2015 IEEE 35th International Conference on Distributed Computing Systems, Columbus, OH, 2015, pp. 732-733.

[2] C. Guo, J. H. Shao, W. Ke and C. Y. Zhang, "A Visible Light Indoor Positioning Algorithm Based on Fingerprint," 2018 4th Annual International Conference on Network and Information Systems for Computers (ICNISC), Wuhan, China, 2018, pp. 71-77.

[3] S. Hamidi-Rad, K. Lyons and N. Goela, "Infrastructureless indoor localization using light fingerprints," 2017 IEEE International

[4] H. Kobayashi, "CEPHEID: the infrastructure-less indoor localization using lighting fixtures' acoustic frequency fingerprints," IECON 2019 - 45th Annual Conference of the IEEE Industrial Electronics Society, Lisbon, Portugal, 2019, pp. 6842-6847.

[5] T. Ince, S. Kiranyaz, L. Eren, M. Askar, and M. Gabbouj, "Real-time motor fault detection by 1-D convolutional neural networks,' IEEE Trans. Ind. Electron., vol. 63, no. 11, pp. 7067-7075, Nov. 2016.

[6] S. Kiranyaz, T. Ince, and M. Gabbouj, "Real-time patientspecific ecg classification by 1-d convolutional neural networks," IEEE Transactions on Biomedical Engineering, vol. 63, no. 3, pp. 664-675, 2016

[7] Sun, Y.; Wang, Y.; Guo, L.; Ma, Z.; Jin, S. The comparison of optimizing SVM by GA and grid search. In Proceedings of the 2017 13th IEEE International Conference on Electronic Measurement \& Instruments (ICEMI), Yangzhou, China, 20-22 October 2017. 